$\exists \int$ Journal of Logic \& Analysis 1:5 (2009) 1-25

ISSN 1759-9008

\title{
Nonstandard Hulls of Locally Exponential Lie Algebras
}

\author{
ISAAC GOLDBRING $^{1}$
}

\begin{abstract}
We show how to construct the nonstandard hull of certain infinitedimensional Lie algebras in order to generalize a theorem of Pestov on the enlargeability of Banach-Lie algebras, yielding a partial answer to a question of Neeb from [11]. In the process, we consider a nonstandard smoothness condition on functions between locally convex spaces to ensure that the induced function between the nonstandard hulls is smooth. We also discuss some conditions on a function between locally convex spaces which guarantee that its nonstandard extension maps finite points to finite points.
\end{abstract}

2000 Mathematics Subject Classification 22E65 (primary); 26E35, 26E20 (secondary)

Keywords: locally exponential Lie group, locally exponential Lie algebra, nonstandard hulls

\section{Introduction}

If $E$ is a Banach space and $M$ is a Hausdorff space, then an $E$-chart on $M$ is a homeomorphism between an open subset of $M$ and an open subset of $E$. The definitions of smooth compatibility of $E$-charts, smooth $E$-atlases on $M$, and smooth $E$-structures on $M$ can be defined exactly as in the finite-dimensional setting, where smoothness for functions between Banach spaces is taken in the sense of Fréchet. A Banach manifold is a Hausdorff space equipped with a smooth $E$-structure for some Banach space $E$. Products of Banach manifolds and smooth functions between Banach manifolds are defined as usual. A Banach-Lie group is a Banach manifold $G$ which is also a group and for which the multiplication map $G \times G \rightarrow G$ and inversion map $G \rightarrow G$ are smooth. As in the finite-dimensional setting, for a Banach-Lie group $G$, one defines $\operatorname{Lie}(G)$ to be the set of left-invariant smooth vector fields on $G$; this is a Banach-space isomorphic to the Banach space that $G$ is modeled on. Moreover, equipped with the Lie bracket of vector fields, $\operatorname{Lie}(G)$ is a Banach-Lie algebra, that is a Banach space equipped with a continuous Lie bracket.

\footnotetext{
${ }^{1}$ This research was supported by an Arnold O. Beckman Research Award.
} 
A Banach-Lie algebra is enlargeable if it is isomorphic to the Lie algebra of a BanachLie group. While all finite-dimensional Lie algebras are enlargeable (this is Lie's third theorem), there are Banach-Lie algebras which are not enlargeable; see, for example, Lazard and Tits [9]. In the early 1990s, Pestov [13] gave a nonstandard hull construction for Banach-Lie algebras and groups and used it to prove the following theorem on the enlargeability of Banach-Lie algebras.

Theorem 1.1 Let $\mathfrak{g}$ be a Banach-Lie algebra. Suppose that there exists a family $\mathcal{H}$ of closed Lie subalgebras of $\mathfrak{g}$ and a neighborhood $V$ of 0 in $\mathfrak{g}$ such that:

- For each $\mathfrak{h}_{1}, \mathfrak{h}_{2} \in \mathcal{H}$, there is an $\mathfrak{h}_{3} \in \mathcal{H}$ such that $\mathfrak{h}_{1} \cup \mathfrak{h}_{2} \subseteq \mathfrak{h}_{3}$;

- $\bigcup \mathcal{H}$ is dense in $\mathfrak{g}$;

- Every $\mathfrak{h} \in \mathcal{H}$ is enlargeable and if $H$ is a corresponding connected, simply connected Lie group, then the restriction $\exp _{H} \mid V \cap \mathfrak{h}$ is injective.

Then $\mathfrak{g}$ is enlargeable.

Nowadays, it is recognized that the setting of Banach-Lie groups and algebras is too restrictive when studying infinite-dimensional Lie groups and algebras and the proper model spaces for such groups and algebras are arbitrary locally convex spaces. Recall that a locally convex space is a Hausdorff topological vector space $E$ for which there is a basis of neighborhoods of 0 consisting of convex sets. (In this paper, all topological vector spaces are assumed to be real.) Equivalently, a locally convex space is a vector space $E$ equipped with a separating family of seminorms; these seminorms yield a topology on $E$ for which a subbase of open sets around 0 are sets of the form

$$
V(p, \epsilon):=\{x \in E \mid p(x)<\epsilon\},
$$

as $p$ varies over the family of seminorms and $\epsilon$ ranges over $\mathbb{R}^{>0}$. After defining smoothness for functions between locally convex spaces, one defines locally convex Lie groups as in the Banach setting above. Locally convex Lie algebras are defined to be locally convex spaces equipped with a continuous Lie bracket. For an introduction to infinite-dimensional Lie theory as it is now studied, see the wonderful survey [11] by Karl-Hermann Neeb. From now on in this paper, when we speak of Lie groups and algebras, we always mean Lie groups and algebras modeled on locally convex spaces.

Since arbitrary Lie groups and algebras lack much of the structure theory of their finitedimensional counterparts, one usually adds extra assumptions on the groups/algebras to be able to develop an adequate Lie theory. A Banach-Lie group is an example of a locally exponential Lie group, which is a Lie group possessing a smooth exponential function which provides a diffeomorphism between an open neighborhood of 0 in its 
Lie algebra and an open neighborhood of the identity in the Lie group. There is a corresponding notion of a locally exponential Lie algebra, which is a Lie algebra that is a natural candidate to be the Lie algebra of a locally exponential Lie group (see Section 5 for precise definitions of these notions). It is one of the open problems in the Neeb survey [11, Problem VI.6] to generalize Pestov's theorem to the class of locally exponential Lie algebras.

Just as in the Banach setting, we can construct the nonstandard hull of an arbitrary internal Lie algebra and prove that it is also a Lie algebra (modeled on the nonstandard hull of the original model space). Whereas the saturation assumption on the nonstandard extension yields quite easily that the nonstandard hull of an internal Banach-Lie algebra is once again a Banach-Lie algebra, it is not at all immediate that the hull of a locally exponential Lie algebra is also a locally exponential Lie algebra. This is due to the fact that locally exponential Lie algebras are defined in terms of smooth functions on the underlying locally convex space, and internally smooth functions do not necessarily induce smooth functions on the nonstandard hull. This is why we have to strengthen the notion of smoothness in our locally exponential Lie algebras to ensure that the nonstandard hull is once again a locally exponential Lie algebra.

In the Banach setting, if an internal Lie algebra is enlargeable, the theory of the BakerCampbell-Hausdorff $(\mathrm{BCH})$ series allows one to construct the nonstandard hull of the corresponding Banach-Lie group in a straightforward manner. We are not as fortunate in our setting, and so our theorem requires an extra (necessary) hypothesis relating the local group operations of the various subalgebras in $\mathcal{H}$ in order to construct the nonstandard hull of an internal Lie group whose Lie algebra is an element of $\mathcal{H}^{*}$.

We do not assume that the reader is familiar with infinite-dimensional Lie theory and so all relevant notions will be defined.

We assume that the reader is familiar with elementary nonstandard analysis; otherwise, consult Davis [3] or Henson [6] for a friendly introduction. Let us say that all nonstandard arguments take place in a sufficiently saturated nonstandard extension.

Here are a few conventions that we use throughout the paper. We always suppose $m$ and $n$ range over $\mathbb{N}:=\{0,1,2, \ldots\}$. For any set $A, A^{\times n}$ denotes the cartesian product $\underbrace{A \times \cdots \times A}_{n \text { times }}$. If $G$ is a group and $A \subseteq G$, then $A^{n}$ denotes the set of $n$-fold products from $A$, that is

$$
A^{n}:=\left\{a_{1} \cdots a_{n} \mid a_{i} \in A \text { for all } i=1, \ldots, n\right\} .
$$

For any topological space $X$ and any $a \in X$, we let

$$
\mu_{X}(a):=\bigcap\left\{\mathcal{O}^{*} \mid \mathcal{O} \text { is an open neighborhood of } a \text { in } X\right\} .
$$


If the space $X$ is clear from context, we write $\mu(a)$ instead of $\mu_{X}(a)$. We also set $X_{\mathrm{ns}}:=\bigcup_{a \in X} \mu(a)$.

Now suppose $X$ is a locally convex space and $\Gamma$ is a set of seminorms defining the topology on $X$. Then for $Y$ an internal subset of $X^{*}$, we define the set

$$
Y_{f}:=\left\{x \in Y \mid p(x) \in \mathbb{R}_{\mathrm{ns}} \text { for all } p \in \Gamma\right\} .
$$

If $Y=X^{*}$, we will just write $X_{f}$ for this set instead of $X_{f}^{*}$. We also set

$$
\mu_{Y}(0):=\left\{x \in Y \mid p(x) \in \mu_{\mathbb{R}}(0) \text { for all } p \in \Gamma\right\}
$$

and we sometimes write $\mu(Y)$ or $\mu_{Y}$ for this set. As before, if $Y=X^{*}$, we just write $\mu(X)$ or $\mu_{X}$, and note that this is equal to $\mu_{X}(0)$ as defined in the previous paragraph. Finally, for $a, b \in X^{*}$, we write $a \sim b$ if $a-b \in \mu(X)$.

I would like to thank Lou van den Dries, Ward Henson, and Karl-Hermann Neeb for very helpful discussions.

\section{Nonstandard Hulls of Internal Lie Algebras}

In this section, we work with the following setting. We let $\mathfrak{g}$ be a locally convex Lie algebra, that is $\mathfrak{g}$ is a locally convex space equipped with a continuous Lie bracket $[\cdot, \cdot]: \mathfrak{g} \times \mathfrak{g} \rightarrow \mathfrak{g}$. We let $\Gamma_{\mathfrak{g}}$ denote the set of all continuous seminorms on $\mathfrak{g}$. We further suppose that $\mathfrak{h}$ is an internal subalgebra of $\mathfrak{g}^{*}$, that is $\mathfrak{h}$ is an internal $\mathbb{R}^{*}$-linear subspace of $\mathfrak{g}$ such that $[\mathfrak{h}, \mathfrak{h}] \subseteq \mathfrak{h}$. Our goal in this section is to form the nonstandard hull of $\mathfrak{h}$.

Lemma 2.1 $\mathfrak{h}_{f}$ is a real Lie algebra and $\mu_{\mathfrak{h}}$ is a Lie ideal of $\mathfrak{h}_{f}$.

Proof It is well-known and easy to see that $\mathfrak{h}_{f}$ is a real vector space and $\mu_{\mathfrak{h}}$ is a real subspace of $\mathfrak{h}_{f}$. We first show that $\left[\mathfrak{h}_{f}, \mathfrak{h}_{f}\right] \subseteq \mathfrak{h}_{f}$. Since $[\cdot, \cdot]$ is continuous at $(0,0)$, given $p \in \Gamma_{\mathfrak{g}}$, there exist $q \in \Gamma_{\mathfrak{g}}$ and $r \in \mathbb{R}^{>0}$ such that for all $a, b \in \mathfrak{g}$, if $q(a), q(b)<r$, then $p([a, b])<1$. Since $x, y \in \mathfrak{h}_{f}$, we can choose $\alpha \in \mathbb{R}^{>0}$ so that $q(\alpha x), q(\alpha y)<r$. Then $p([\alpha x, \alpha y])<1$, whence $p([x, y])<\frac{1}{\alpha^{2}}$. It follows that $[x, y] \in \mathfrak{h}_{f}$ and hence $\mathfrak{h}_{f}$ is a real Lie algebra.

It remains to show that $\left[\mathfrak{h}_{f}, \mu_{\mathfrak{h}}\right] \subseteq \mu_{\mathfrak{h}}$. Suppose $x \in \mathfrak{h}_{f}$ and $y \in \mu_{\mathfrak{h}}$. Let $p \in \Gamma_{\mathfrak{g}}$ and let $\epsilon \in \mathbb{R}^{>0}$. By continuity of $[\cdot, \cdot]$ at $(0,0)$, there exists $q \in \Gamma_{\mathfrak{g}}$ and $r \in \mathbb{R}^{>0}$ such that for all $a, b \in \mathfrak{g}$, if $q(a), q(b)<r$, then $p([a, b])<\epsilon$. Since $x \in \mathfrak{h}_{f}$, we can choose $\alpha \in \mathbb{R}^{>0}$ so that $q(\alpha x)<r$. Since $y \in \mu_{\mathfrak{h}}$, we have that $q\left(\frac{1}{\alpha} y\right)<r$, whence we can conclude that $p([x, y])=p\left(\left[\alpha x, \frac{1}{\alpha} y\right]\right)<\epsilon$. But $\left[\alpha x, \frac{1}{\alpha} y\right]=[x, y]$, whence we see that $p([x, y])<\epsilon$. Since $p$ and $\epsilon$ were arbitrary, we see that $[x, y] \in \mu_{\mathfrak{h}}$. 
Define the nonstandard hull of $\mathfrak{h}$ to be $\widehat{\mathfrak{h}}:=\mathfrak{h}_{f} / \mu_{\mathfrak{h}}$, which is a real Lie algebra. (The Lie bracket is given by $\left[x+\mu_{\mathfrak{h}}, y+\mu_{\mathfrak{h}}\right]:=[x, y]+\mu_{\mathfrak{h}}$.) Let $\pi_{\mathfrak{h}}: \mathfrak{h}_{f} \rightarrow \widehat{\mathfrak{h}}$ denote the canonical quotient map. For $p \in \Gamma_{\mathfrak{g}}$, define $\widehat{p}: \widehat{\mathfrak{h}} \rightarrow \mathbb{R}$ by $\widehat{p}\left(x+\mu_{\mathfrak{h}}\right):=\operatorname{st}(p(x))$; we can make this definition because for all $x, y \in \mathfrak{g},|p(x)-p(y)| \leq p(x-y)$.)

Let $\Gamma_{\widehat{\mathfrak{h}}}:=\left\{\widehat{p} \mid p \in \Gamma_{\mathfrak{g}}\right\}$. We claim that $\Gamma_{\widehat{\mathfrak{h}}}$ is a separating family of seminorms on $\widehat{\mathfrak{h}}$. It is trivial to verify that each $\widehat{p}$ is a seminorm. To see that $\Gamma_{\widehat{h}}$ is separating, note that if $x \in \mathfrak{h}_{f} \backslash \mu_{\mathfrak{h}}$, then for some $p \in \Gamma_{\mathfrak{g}}$ and some $\epsilon \in \mathbb{R}^{>0}, p(x) \geq \epsilon$. Consequently, $\widehat{p}\left(x+\mu_{\mathfrak{h}}\right) \geq \epsilon$.

We now see that $\widehat{\mathfrak{h}}$ equipped with the family of seminorms $\Gamma_{\widehat{\mathfrak{h}}}$ is a locally convex space. It remains to show that the Lie bracket of $\widehat{\mathfrak{h}}$ is continuous with respect to the locally convex topology just given to $\widehat{\mathfrak{h}}$.

Lemma $2.2[\cdot, \cdot]: \widehat{\mathfrak{h}} \times \widehat{\mathfrak{h}} \rightarrow \widehat{\mathfrak{h}}$ is continuous and thus $\widehat{\mathfrak{h}}$ is a locally convex Lie algebra.

Proof We first show the continuity of $[\cdot, \cdot]$ at $\left(0+\mu_{\mathfrak{h}}, 0+\mu_{\mathfrak{h}}\right)$. Let $\widehat{p} \in \Gamma_{\widehat{\mathfrak{h}}}$ and let $\epsilon \in \mathbb{R}^{>0}$. Fix $\epsilon^{\prime} \in \mathbb{R}^{>0}$ with $\epsilon^{\prime}<\epsilon$. Choose $q \in \Gamma_{\mathfrak{g}}$ and $r \in \mathbb{R}^{>0}$ so that if $a, b \in \mathfrak{g}$ and $q(a), q(b)<r$, then $p([a, b])<\epsilon^{\prime}$. Then if $\widehat{q}\left(x+\mu_{\mathfrak{h}}\right), \widehat{q}\left(y+\mu_{\mathfrak{h}}\right)<r$, one has $\widehat{p}\left([x, y]+\mu_{\mathfrak{h}}\right) \leq \epsilon^{\prime}<\epsilon$.

We next show that for any $c+\mu_{\mathfrak{h}} \in \widehat{\mathfrak{h}}$, the map

$$
x+\mu_{\mathfrak{h}} \mapsto[c, x]+\mu_{\mathfrak{h}}: \widehat{\mathfrak{h}} \rightarrow \widehat{\mathfrak{h}}
$$

is continuous at $0+\mu_{\mathfrak{h}}$ (and hence continuous on all of $\widehat{\mathfrak{h}}$ since the aforementioned map is linear). Fix $\widehat{p} \in \Gamma_{\widehat{\mathfrak{h}}}$ and $\epsilon \in \mathbb{R}^{>0}$. Fix $\epsilon^{\prime} \in \mathbb{R}^{>0}$ with $\epsilon^{\prime}<\epsilon$. As in the above paragraph, choose $q \in \Gamma_{\mathfrak{g}}$ and $r \in \mathbb{R}^{>0}$ so that if $a, b \in \mathfrak{g}$ satisfy $q(a), q(b)<r$, then $p([a, b])<\epsilon^{\prime}$. Choose $\alpha \in \mathbb{R}^{>0}$ so that $q(\alpha c)<r$. Now suppose $\widehat{q}\left(x+\mu_{\mathfrak{h}}\right)<\alpha r$. Then $q\left(\frac{1}{\alpha} x\right)<r$, whence $p\left(\left[\alpha c, \frac{1}{\alpha} x\right]\right)<\epsilon^{\prime}$. Thus, $\widehat{p}\left([c, x]+\mu_{\mathfrak{h}}\right)<\epsilon$.

An analogous argument shows that for any $c+\mu_{\mathfrak{h}} \in \widehat{\mathfrak{h}}$, the map

$$
x+\mu_{\mathfrak{h}} \mapsto[x, c]+\mu_{\mathfrak{h}}: \widehat{\mathfrak{h}} \rightarrow \widehat{\mathfrak{h}}
$$

is also continuous. We can thus conclude that $[\cdot, \cdot]: \widehat{\mathfrak{h}} \rightarrow \widehat{\mathfrak{h}}$ is continuous from the fact that for a topological vector space $X$, a bilinear map $T: X \times X \rightarrow X$ is continuous if it is continuous at $\left(0_{X}, 0_{X}\right)$ and if for each $a \in X$, the functions $x \mapsto T(a, x): X \rightarrow X$ and $x \mapsto T(x, a): X \rightarrow X$ are continuous. (This is probably well known, but here is a nonstandard proof of this. Suppose $(a, b) \in X \times X$ and $(c, d) \in \mu(a, b)$. Then

$$
T(a, b)-T(c, d)=T(a-c, b)+T(c-a, b-d)+T(a, b-d),
$$

which is in $\mu(X)$ by our assumptions.) 
Remark 2.3 It is obvious that the linear map $\iota: \mathfrak{g} \rightarrow \widehat{\mathfrak{g}^{*}}$ given by $\iota(x)=x+\mu$ is such that for every $p \in \Gamma_{\mathfrak{g}}$ and every $x \in \mathfrak{g}$, one has $p(x)=\widehat{p}(\iota(x))$. In particular, $\iota$ is an injective morphism of locally convex Lie algebras, that is a continuous Lie algebra homomorphism.

Remark 2.4 An easy saturation argument shows that $\widehat{\mathfrak{h}}$ is a closed subspace of $\widehat{\mathfrak{g}^{*}}$. Since $\widehat{\mathfrak{g}}^{*}$ is complete (see Luxemberg [10, Theorem 3.15.1]), it follows that $\widehat{\mathfrak{h}}$ is complete as well.

\section{Nonstandard Differentiability Conditions in Locally Con- vex Spaces}

In this section, we define a nonstandard notion of smoothness for functions between locally convex spaces which is stronger than the standard notion of smoothness and show how such functions induce (standardly) smooth functions on the nonstandard hulls. We then introduce a standard strengthening of smoothness which implies our nonstandard notion. Finally, we show that for certain locally convex spaces, our nonstandard notion is equivalent to ordinary smoothness.

Throughout this section, we assume $E$ and $F$ are locally convex spaces, $U \subseteq E$ is open, and $f: U \rightarrow F$ is a function. Before we enter our discussion of differentiability, we first provide the following easy lemma, which may be well-known but is included here for the sake of completeness. Using the terminology of Stroyan [14], we let $\operatorname{Lin}^{k}\left(E^{*}, F^{*}\right)$ denote the space of internal $k$-linear maps from $E^{*}$ to $F^{*}$ and we introduce the space

$$
\operatorname{FLin}^{k}\left(E^{*}, F^{*}\right)=\left\{T \in \operatorname{Lin}^{k}\left(E^{*}, F^{*}\right) \mid T\left(\left(E_{f}\right)^{\times k}\right) \subseteq F_{f}\right\} .
$$

We let $\operatorname{FLin}\left(E^{*}, F^{*}\right)$ denote $\operatorname{FLin}^{1}\left(E^{*}, F^{*}\right)$.

Lemma 3.1 Suppose $T \in \operatorname{Lin}^{k}\left(E^{*}, F^{*}\right)$. Then $T \in \operatorname{FLin}^{k}\left(E^{*}, F^{*}\right)$ if and only if whenever $x_{1}, \ldots, x_{k} \in E_{f}$ are such that $x_{i} \in \boldsymbol{\mu}_{E}$ for some $i \in\{1, \ldots, k\}$, we have $T\left(x_{1}, \ldots, x_{k}\right) \in \mu_{F}$.

Proof First suppose that $T \in \operatorname{FLin}^{k}\left(E^{*}, F^{*}\right)$. Let $x_{1}, \ldots, x_{k} \in E_{f}$ and assume, without loss of generality, that $x_{1} \in \mu_{E}$. Choose $N \in \mathbb{N}^{*} \backslash \mathbb{N}$ such that $N x_{1} \in E_{f}$; such $N$ exists by an easy saturation argument (see Henson and Moore [7, Theorem 1.6]). But now

$$
T\left(x_{1}, \ldots, x_{k}\right)=\frac{1}{N} T\left(N x_{1}, x_{2}, \ldots, x_{k}\right) \in \frac{1}{N} F_{f} \subseteq \mu_{F} .
$$


We now prove the reverse implication. Suppose that there are $x_{1}, \ldots, x_{k} \in E_{f}$ for which $T\left(x_{1}, \ldots, x_{k}\right) \notin F_{f}$. We can then find a continuous seminorm $p$ on $F$ such that $N:=p\left(T\left(x_{1}, \ldots, x_{k}\right)\right) \in \mathbb{N}^{*} \backslash \mathbb{N}$. But then

$$
p\left(T\left(\frac{1}{N} x_{1}, x_{2}, \ldots, x_{k}\right)\right)=1,
$$

whence we conclude that $T\left(\frac{1}{N} x_{1}, x_{2}, \ldots, x_{k}\right) \notin \mu_{F}$.

We now recall the (standard) notion of smoothness that appears in the Neeb survey [11] and the stronger (nonstandard) notion defined by Stroyan in [14].

Definition 3.2 Let $a \in U$. Then $f$ is differentiable at $a$ if for all $h \in E$, the limit

$$
\lim _{t \rightarrow 0} \frac{1}{t}(f(a+t h)-f(a))
$$

exists. We denote this limit by $d f(a)(h)$ or $D_{h} f(a)$. We say that $f$ is differentiable if $f$ is differentiable at $a$ for all $a \in U$. We say that $f$ is $C^{1}$ if $f$ is differentiable and the map $d f: U \times E \rightarrow F$ is continuous. $f$ is said to be $C^{k}$ if it is continuous, the iterated directional derivatives

$$
d^{j} f(a)\left(h_{1}, \ldots, h_{j}\right):=\left(D_{h_{j}} \cdots D_{h_{1}} f\right)(a)
$$

exist for all $j \in\{1, \ldots, k\}, a \in U$, and $h_{1}, \ldots, h_{j} \in E$ and all maps $d^{j} f: U \times E^{j} \rightarrow F$ are continuous. Finally, we say that $f$ is smooth if $f$ is $C^{k}$ for all $k$.

Notation: If $U$ is an open subset of $E$, we let

$$
\operatorname{in}\left(U^{*}\right)=\left\{a \in U^{*} \mid \text { for all } b \in E^{*}, \text { if } b \sim a \text {, then } b \in U^{*}\right\} .
$$

Definition 3.3 (Stroyan [14]) $f$ is uniformly differentiable if there is a map

$$
d f: U \rightarrow \operatorname{Lin}(E, F)
$$

such that for every $a \in \operatorname{in}\left(U^{*}\right) \cap E_{\mathrm{ns}}$, one has $d f(a) \in \operatorname{FLin}\left(E^{*}, F^{*}\right)$, and for every $h \in E_{f}$ and for every positive $\delta \in \mu(\mathbb{R})$, we have

$$
\frac{1}{\delta}(f(a+\delta h)-f(a)) \sim d f(a)(h) .
$$

The notion $f$ is uniformly $C^{k}$ is defined recursively as follows. $f$ is uniformly $C^{1}$ means $f$ is uniformly differentiable. Suppose $f$ is uniformly $C^{k}$. Then we say $f$ is uniformly $C^{k+1}$ if there is a map $d^{k+1} f: U \rightarrow \operatorname{Lin}^{k+1}(E, F)$ so that whenever $a \in \operatorname{in}\left(U^{*}\right) \cap E_{\mathrm{ns}}$, then $d^{k+1} f(a) \in \operatorname{FLin}^{k+1}\left(E^{*}, F^{*}\right)$, and whenever $x \in E_{f}, h \in\left(E_{f}\right)^{\times k}$, and $\delta \in \mu(\mathbb{R})$ is positive, we have

$$
\frac{1}{\delta}\left(d^{k} f(a+\delta x)(h)-d^{k} f(a)(h)\right) \sim d^{k+1} f(a)(h, x) .
$$

We say that $f$ is uniformly smooth if $f$ is uniformly $C^{k}$ for every $k$. 
The notion of being uniformly $C^{k}$ is really a strengthening of the notion of being $C^{k}$.

Lemma 3.4 Suppose $f$ is uniformly $C^{k}$. Then $f$ is $C^{k}$.

Proof For simplicity, we only prove this for the case $k=1$, the higher order cases being similar. The assumption of uniformly differentiable clearly implies that $f$ is differentiable. What is left to show is the map $d f: U \times E \rightarrow F$ is continuous. Suppose $a \in U, a^{\prime} \in \mu(a), h \in E, h^{\prime} \in \mu(h)$. We must show $d f(a)(h) \sim d f\left(a^{\prime}\right)\left(h^{\prime}\right)$. By Lemma 3.1, we know $d f(a)(h) \sim d f(a)\left(h^{\prime}\right)$. By Stroyan [14, Proposition 2.4], $d f(a)\left(h^{\prime}\right) \sim d f\left(a^{\prime}\right)\left(h^{\prime}\right)$. This completes the proof.

For our purposes, we will need the following strengthening of Stroyan's definition.

Definition 3.5 $f$ is uniformly differentiable at finite points if there is a map

$$
d f: U \rightarrow \operatorname{Lin}(E, F)
$$

such that, for every $a \in \operatorname{in}\left(U^{*}\right) \cap E_{f}$, one has $d f(a) \in \operatorname{FLin}\left(E^{*}, F^{*}\right)$, and for every $h \in E_{f}$ and for every positive $\delta \in \mu(\mathbb{R})$, we have

$$
\frac{1}{\delta}(f(a+\delta h)-f(a)) \sim d f(a)(h) .
$$

Example 3.6 (Stroyan [14]) Let $E=F=\mathbb{R}^{\mathbb{N}}$ be given its usual structure as a locally convex space, that is the topology is generated by the seminorms $p_{j}(j \in \mathbb{N})$, where, for $a=\left(a_{i}\right) \in E, p_{j}(a):=\max \left\{\left|a_{1}\right|, \ldots,\left|a_{j}\right|\right\}$. Let $f: E \rightarrow F$ be the map $f(a)=\left(\sin \left(i a_{i}\right)\right)$. Let $a, x \in E_{f}$ (that is $a_{i}, x_{i} \in \mathbb{R}_{f}$ for $i \in \mathbb{N}$ ). Then

$$
\begin{aligned}
\left(\frac{1}{\delta}((f(a+\delta x)-f(a)))_{i}\right. & =\sin \left(i a_{i}\right) \frac{\cos \left(i x_{i}\right)-1}{\delta}+\cos \left(i a_{i}\right) \frac{\sin \left(i \delta x_{i}\right)}{\delta} \\
& =i x_{i} \cos \left(i a_{i}\right)+\delta \cdot z_{i}
\end{aligned}
$$

where $z_{i} \in \mathbb{R}_{f}$. By defining $d f: E \rightarrow \operatorname{Lin}(E, F)$ by $d f(a)(x)(i):=i \cos \left(i a_{i}\right) x_{i}$, we see that $f$ is uniformly differentiable at finite points.

Lemma 3.7 Suppose $f$ is uniformly differentiable at finite points. Then $f$ is $S$ continuous at finite points, that is if $w \in \operatorname{in}\left(U^{*}\right) \cap E_{f}$ and $z \sim w$, then $f(w) \sim f(z)$. 
Proof Fix $w$ and $z$ as in the statement of the lemma. Again, by Henson and Moore [7, Theorem 1.6], there exists $N \in \mathbb{N}^{*} \backslash \mathbb{N}$ such that $x:=N(w-z) \in \mu(E)$. Let $\delta:=\frac{1}{N}$. By uniform differentiability at $z$, there is $\eta \in \mu(F)$ such that

$$
\frac{1}{\delta}[f(z+\delta x)-f(z)]=d f(z)(x)+\eta,
$$

that is

$$
\frac{1}{\delta}[f(w)-f(z)]=d f(z)(N(w-z))+\eta .
$$

Hence $f(w)-f(z)=d f(z)(w-z)+\delta \cdot \eta \in \mu(F)$ by Lemma 3.1.

Lemma 3.8 If $f$ is uniformly differentiable at finite points, then $d f$ is $S$-continuous at finite points, that is if $a, a^{\prime} \in \operatorname{in}\left(U^{*}\right) \cap E_{f}$ are such that $a \sim a^{\prime}$, and $x, x^{\prime} \in E_{f}$ are such that $x \sim x^{\prime}$, then $d f(a)(x) \sim d f\left(a^{\prime}\right)\left(x^{\prime}\right)$.

Proof One shows that $d f(a)(x) \sim d f\left(a^{\prime}\right)(x)$ exactly as the proof of Stroyan [14, Proposition 2.4]. Then, since $d f\left(a^{\prime}\right) \in \operatorname{FLin}\left(E^{*}, F^{*}\right)$, one has $d f\left(a^{\prime}\right)(x) \sim d f\left(a^{\prime}\right)\left(x^{\prime}\right)$ by Lemma 3.1.

Notation: In the rest of this paper, for any locally convex space $E$, any internal $\mathbb{R}^{*}$ linear subspace $Y$ of $E^{*}$, and any $x \in Y_{f}$, we may denote the element $x+\mu(Y)$ of $\widehat{Y}$ by $\llbracket x \rrbracket$.

For the rest of this subsection, let us assume that $f\left(U^{*} \cap E_{f}\right) \subseteq F_{f}$. (We will take up the issue of when this happens in the next section.) Since $U$ is open, we can write

$$
U=\bigcup_{i \in I} \bigcap_{j=1}^{n_{i}}\left\{x \in E \mid p_{i j}\left(x-x_{i j}\right)<\epsilon_{i j}\right\},
$$

for some continuous seminorms $p_{i j}$ on $E$, some elements $x_{i j} \in E$ and some $\epsilon_{i j} \in \mathbb{R}^{>0}$. Let us then define

$$
\widehat{U}:=\bigcup_{i \in I} \bigcap_{j=1}^{n_{i}}\left\{\llbracket x \rrbracket \in \widehat{E} \mid \widehat{p_{i j}}\left(\llbracket x \rrbracket-\llbracket x_{i j} \rrbracket\right)<\epsilon_{i j}\right\} .
$$

It is clear that $\widehat{U}$ is an open subset of $\widehat{E}$ and that if $\llbracket x \rrbracket \in \widehat{U}$, then $x \in \operatorname{in}\left(U^{*}\right) \cap E_{f}$. If we further assume that $f$ is $S$-continuous at finite points (in particular if $f$ is uniformly differentiable at finite points), then we get a continuous map $\widehat{f}: \widehat{U} \rightarrow \widehat{F}$ given by $\widehat{f}(\llbracket a \rrbracket)=\llbracket f(a) \rrbracket$.

Proposition 3.9 Suppose $f$ is uniformly differentiable at finite points. Then $\widehat{f}$ is $C^{1}$. 
Proof By Lemma 3.8, we can define the map

$$
\widehat{d f}: \widehat{U} \times \widehat{E} \rightarrow \widehat{F}, \quad \widehat{d f}(\llbracket a \rrbracket, \llbracket h \rrbracket)=\llbracket d f(a)(h) \rrbracket .
$$

We now show, for $\llbracket a \rrbracket \in \widehat{U}$, that $\widehat{d f}(\llbracket a \rrbracket)$ is the derivative of $\widehat{f}$ at $\llbracket a \rrbracket$. In order to do this, let $\llbracket h \rrbracket \in \widehat{E}, \widehat{p}$ a continuous seminorm on $\widehat{E}$ and $\epsilon \in \mathbb{R}^{>0}$. We need a $\delta \in \mathbb{R}^{>0}$ so that if $|t|<\delta$, then

$$
\left.\widehat{p}\left(\llbracket d f(a)(h) \rrbracket-\frac{1}{t} \widehat{f}(\llbracket a \rrbracket+t \llbracket h \rrbracket)-\widehat{f}(\llbracket a \rrbracket)\right)\right)<\epsilon,
$$

that is we need a $\delta \in \mathbb{R}^{>0}$ so that if $|t|<\delta$, then

$$
\operatorname{st}\left(p\left(d f(a)(h)-\frac{1}{t}(f(a+t h)-f(a))\right)\right)<\epsilon .
$$

Since the above expression is 0 if $t$ is infinitesimal (by uniform differentiability at finite points), we can find the desired $\delta$ by saturation.

It remains to show $\widehat{d f}: \widehat{U} \times \widehat{E} \rightarrow \widehat{F}$ is continuous. Fix $[a] \in \widehat{U}$ and $[h] \in \widehat{E}$. Let $\widehat{p}$ be a continuous seminorm on $\widehat{F}$ and $\epsilon \in \mathbb{R}^{>0}$. We need $r \in \mathbb{R}^{>0}$ and continuous seminorms $\widehat{p_{1}}, \ldots, \widehat{p_{n}}$ on $\widehat{E}$ so that if $\widehat{p_{i}}\left([a]-\left[a^{\prime}\right]\right), \widehat{p_{i}}\left([h]-\left[h^{\prime}\right]\right) \leq r$ for $i=1, \ldots, n$, then $\widehat{p}\left(\widehat{d f}([a])([h])-\widehat{d f}\left(\left[a^{\prime}\right]\right)\left(\left[h^{\prime}\right]\right)\right)<\epsilon$. If not, then one can use saturation to get $a^{\prime} \in U^{*}$ with $a^{\prime} \sim a$ and $h^{\prime} \in E_{f}$ with $h^{\prime} \sim h$ such that $p\left(d f(a)(h)-d f\left(a^{\prime}\right)\left(h^{\prime}\right)\right) \geq \epsilon$, which contradicts the S-continuity of $d f$ at finite points.

Definition 3.10 The notion $f$ is uniformly $C^{k}$ at finite points is defined recursively as follows. $f$ is uniformly $C^{1}$ at finite points means $f$ is uniformly differentiable at finite points. Suppose $f$ is uniformly $C^{k}$ at finite points. Then $f$ is uniformly $C^{k+1}$ at finite points if there is a map $d^{k+1} f: U \rightarrow \operatorname{Lin}^{k+1}(E, F)$ so that whenever $a \in \operatorname{in}\left(U^{*}\right) \cap E_{f}$, we have $d^{k+1} f(a) \in \operatorname{FLin}^{k+1}\left(E^{*}, F^{*}\right)$ and whenever $x \in E_{f}, h \in\left(E_{f}\right)^{\times k}$, and $\delta \in \mu(\mathbb{R})$ is positive, we have

$$
\frac{1}{\delta}\left(d^{k} f(a+\delta x)(h)-d^{k} f(a)(h)\right) \sim d^{k+1} f(a)(h, x) .
$$

We will say that $f$ is uniformly smooth at finite points if $f$ is uniformly $C^{k}$ at finite points for every $k \geq 1$.

Proposition 3.11 If $f$ is uniformly $C^{k}$ at finite points, then $\widehat{f}$ is $C^{k}$ and

$$
d^{k} \widehat{f}(\llbracket a \rrbracket)\left(\llbracket h_{1} \rrbracket, \ldots, \llbracket h_{k} \rrbracket\right)=\llbracket d^{k} f(a)\left(h_{1}, \ldots, h_{k}\right) \rrbracket .
$$

In particular, if $f$ is uniformly smooth at finite points, then $\widehat{f}$ is smooth. 
Proof By induction on $k$. The case $k=1$ is exactly Proposition 3.9 (and its proof). We now suppose that $f$ is uniformly $C^{k+1}$ at finite points. Fix $\llbracket a \rrbracket \in \widehat{U}$ and $\llbracket h_{1} \rrbracket, \ldots, \llbracket h_{k+1} \rrbracket \in \widehat{E}$. We must show that

$$
d^{k+1} \widehat{f}(\llbracket a \rrbracket)\left(\llbracket h_{1} \rrbracket, \ldots, \llbracket h_{k} \rrbracket\right)=\llbracket d^{k+1} f(a)\left(h_{1}, \ldots, h_{k+1}\right) \rrbracket .
$$

We first must show that the above expression is well-defined. Suppose $a^{\prime} \sim a$ and $h_{i}^{\prime} \sim h_{i}$ for $i=1, \ldots, k+1$. By the analog of Stroyan [14, Proposition 3.2], we know that $d^{k+1} f(a)\left(h_{1}, \ldots, h_{k+1}\right) \sim d^{k+1}\left(a^{\prime}\right)\left(h_{1}, \ldots, h_{k+1}\right)$. However, using the fact that $d f\left(a^{\prime}\right) \in \operatorname{FLin}^{k+1}\left(E^{*}, F^{*}\right)$, Lemma 3.1 shows that

$$
d f\left(a^{\prime}\right)\left(h_{1}, \ldots, h_{k+1}\right) \sim d f\left(a^{\prime}\right)\left(h_{1}^{\prime}, \ldots, h_{k+1}^{\prime}\right) .
$$

For ease of notation, let $h=\left(h_{1}, \ldots, h_{k}\right)$ and $d_{h}^{k} \widehat{f}(\cdot):=d^{k} \widehat{f}(\cdot)\left(h_{1}, \ldots, h_{k}\right)$. We now must show that

$$
\lim _{t \rightarrow 0} \frac{1}{t}\left(d_{h}^{k} \widehat{f}\left(\llbracket a \rrbracket+t \llbracket h_{k+1}\right] \rrbracket-d_{h}^{k} \widehat{f}(\llbracket a \rrbracket)\right)=\llbracket d^{k+1} f(a)\left(h_{1}, \ldots, h_{k+1}\right) \rrbracket .
$$

By induction, this amounts to showing that

$$
\lim _{t \rightarrow 0} \frac{1}{t} \llbracket d^{k} f\left(a+t h_{k+1}\right)(h)-d^{k} f(a)(h) \rrbracket=\llbracket d^{k+1} f(a)\left(h_{1}, \ldots, h_{k+1}\right) \rrbracket .
$$

Let $\widehat{p}$ be a continuous seminorm on $\widehat{F}$ and let $\epsilon \in \mathbb{R}^{>0}$. We need a $\delta \in \mathbb{R}^{>0}$ so that if $|t|<\delta$, then

$$
\operatorname{st}\left(p\left(\frac{1}{t}\left(d^{k} f\left(a+t h_{k+1}\right)-d^{k} f(a)(h)\right)-d^{k+1} f(a)\left(h_{1}, \ldots, h_{k+1}\right)\right)\right)<\epsilon .
$$

Since the above quantity is 0 for infinitesimal $t$, the desired $\delta$ can be obtained by saturation.

Our final obligation is to show that $d^{k+1} \widehat{f}: \widehat{U} \times \widehat{E}^{k+1} \rightarrow \widehat{F}$ is continuous. The proof is identical to the corresponding part of the proof of Proposition 3.9.

\section{Strong Smoothness}

We now introduce a standard condition on $f$ which implies that it is uniformly differentiable at finite points. We first mention some facts from the calculus of locally convex spaces. Suppose $f$ is $C^{1}$. Let

$$
U^{[1]}:=\{(x, y, t) \in U \times E \times \mathbb{R} \mid x+t y \in U\},
$$

an open subset of $E \times E \times \mathbb{R}$. Let $f^{[1]}: U^{[1]} \rightarrow F$ be defined by

$$
f^{[1]}(x, y, t)= \begin{cases}\frac{1}{t}(f(x+t y)-f(x)) & \text { if } t \neq 0 \\ d f(x)(y) & \text { if } t=0\end{cases}
$$


It follows from the Mean Value Theorem (see Neeb [11, Proposition I.2.3]) that $f^{[1]}$ is continuous. In fact, it is shown in Bertram et al [1] that if $f$ is continuous and there exists a continuous function $f^{[1]}: U^{[1]} \rightarrow F$ such that $f^{[1]}(x, y, t)=\frac{1}{t}(f(x+t y)-f(x))$ for $t \neq 0$, then $f$ is $C^{1}$ and $d f(x)(y)=f^{[1]}(x, y, 0)$.

Definition 3.12 Suppose $f$ is $C^{1}$. Then $f$ is strongly $C^{1}$ if $f^{[1]}$ is uniformly continuous.

Lemma 3.13 Suppose $f$ is strongly $C^{1}$. Then $f$ is uniformly differentiable at finite points.

Proof Suppose $a \in \operatorname{in}\left(U^{*}\right) \cap E_{f}$. We first show $d f(a) \in \operatorname{FLin}\left(E^{*}, F^{*}\right)$. It suffices to show that if $x \in \mu(E)$, then $d f(a)(x) \in \mu(F)$. But

$$
d f(a)(x)=f^{[1]}(a, x, 0) \sim f^{[1]}(a, 0,0)=d f(a)(0)=0
$$

since $d f(a)$ is an internal linear map.

Now suppose $x \in E_{f}$ and $\delta$ is a positive element of $\mu(\mathbb{R})$. We must show that $f^{[1]}(a, x, \delta) \sim d f(a)(x)$. But $f^{[1]}(a, x, \delta) \sim f^{[1]}(a, x, 0)=d f(a)(x)$, finishing the proof.

Remark 3.14 Notice that we never used the fact that $a$ and $x$ were finite in the above proof, so being strongly $C^{1}$ implies uniform differentiability at all points and where we are allowed to take derivatives in the direction of any element of $E^{*}$.

Lemma 3.15 If $f$ is strongly $C^{1}$, then $f$ is uniformly continuous.

Proof Suppose $x, y \in U^{*}$ are such that $x \sim y$. Choose $N \in \mathbb{N}^{*} \backslash \mathbb{N}$ such that $z:=N(x-y) \in \mu(E)$. Let $\delta:=\frac{1}{N}$. Then

$$
f(x)-f(y)=f(y+\delta z)-f(y) \sim \delta d f(y)(z)=d f(y)(\delta z) \in \mu(F) .
$$

We now describe the higher order analogs of this notion. One can recursively define the sets $U^{[k]}$ for $k \in \mathbb{N}$ by $U^{[k+1]}:=\left(U^{[k]}\right)^{[1]}$ and the functions $f^{[k]}: U^{[k]} \rightarrow F$ by $f^{[k+1]}:=\left(f^{[k]}\right)^{[1]}$. It is shown in Bertram et al [1] that a $C^{k}$ function $f$ is $C^{k+1}$ if and only if $f^{[k]}$ is $C^{1}$.

Definition 3.16 The notion $f$ is strongly $C^{k}$ is defined recursively as follows. The notion $f$ is strongly $C^{1}$ has already been defined. Assume $f$ is $C^{k+1}$ and strongly $C^{k}$. Then $f$ is strongly $C^{k+1}$ if $f^{[k]}$ is strongly $C^{1}$. We will say that $f$ is strongly smooth if $f$ is strongly $C^{k}$ for all $k \geq 1$. 
Lemma 3.17 If $f$ is strongly $C^{k}$, then $f$ is uniformly $C^{k}$ at finite points.

Proof By induction on $k$. The case $k=1$ is precisely Lemma 3.13. We now assume the result holds for $k$ and we suppose $f$ is strongly $C^{k+1}$. The induction hypothesis gives us that $f$ is uniformly $C^{k}$ at finite points. In order to prove the other two conditions for $f$ to be uniformly $C^{k+1}$ at finite points, we need to elaborate on the relationship between the functions $d^{n} f$ and $f^{[n]}$ for arbitrary $n$.

Using the terminology from Bertram et al [1], each $d^{n} f$ is a partial map of $f^{[n]}$ in the sense that each $d^{n} f$ is obtained from $f^{[n]}$ by fixing some coordinates of the domain of $f^{[n]}$. For example,

$$
d f(x)(h)=f^{[1]}(x, h, 0)
$$

and

$$
d^{2} f(x)\left(h_{1}, h_{2}\right)=f^{[2]}\left(x, h_{1}, 0, h_{2}, 0,0,0\right) .
$$

Hence, if $f^{[n]}$ is uniformly continuous, then so is $d^{n} f$.

Let us show that for any $a \in \operatorname{in}\left(U^{*}\right) \cap E_{f}$, we have $d f(a) \in \operatorname{FLin}^{k+1}(E, F)$. Suppose $h_{1}, \ldots, h_{k+1} \in E_{f}$ and, without loss of generality, that $h_{1} \in \mu(E)$. Then by uniform continuity of $d^{k+1} f$, we have $d^{k+1} f(a)\left(h_{1}, \ldots, h_{k+1}\right) \sim d^{k+1} f(a)\left(0, h_{2} \ldots, h_{k+1}\right)=0$. Now suppose that $h_{1}, \ldots, h_{k}, x \in E_{f}$ and $\delta$ is a positive element of $\mu(\mathbb{R})$. We now show that

$$
\frac{1}{\delta}\left(d^{k} f(a+\delta x)\left(h_{1}, \ldots, h_{k}\right)-d^{k} f(a)\left(h_{1}, \ldots, h_{k}\right)\right) \sim d^{k+1} f(a)\left(h_{1}, \ldots, h_{k}, x\right) .
$$

This follows from the uniform continuity of $f^{[k+1]}$. Let us illustrate this in the case when $k=2$, as the formula relating $d^{k} f$ and $f^{[k]}$ is simple enough in this case. For simplicity, let us denote the left hand side of the above equation by LHS.

$$
\begin{aligned}
\text { LHS } & =\frac{1}{\delta}\left(f^{[2]}\left(a+\delta x, h_{1}, 0, h_{2}, 0,0,0\right)-f^{[2]}\left(a, h_{1}, 0, h_{2}, 0,0,0\right)\right) \\
& =f^{[3]}\left(\left(a, h_{1}, 0, h_{2}, 0,0,0\right),(x, 0,0,0,0,0,0), \delta\right) \\
& \sim f^{[3]}\left(\left(a, h_{1}, 0, h_{2}, 0,0,0\right),(x, 0,0,0,0,0,0), 0\right) \\
& =d^{3} f(a)\left(h_{1}, h_{2}, x\right)
\end{aligned}
$$

\section{The Case of Complete HM-spaces}

Recall that $x \in E^{*}$ is called pre-nearstandard if for every neighborhood $V$ of 0 in $E$, there is $y \in E$ such that $x-y \in V^{*}$. Let $E_{\mathrm{pns}}$ denote the set of pre-nearstandard points 
of $E$ and note that we always have the inclusions $E_{\mathrm{ns}} \subseteq E_{\mathrm{pns}} \subseteq E_{f}$. The importance of the pre-nearstandard points of $E$ is that their image in $\widehat{E}$ is the completion of $E$ in $\widehat{E}$ (so $E$ is complete if and only if $E_{\mathrm{ns}}=E_{\mathrm{pns}}$ ). An HM-space is a locally convex space $E$ for which $E_{\mathrm{pns}}=E_{f}$.

\section{Remarks 3.18}

(1) In standard language, a locally convex space $E$ is an HM-space if and only if whenever $\mathcal{F}$ is an ultrafilter on $E$ with the property that for every $U$ from a fixed neighborhood base of 0 in $E$ there is $n$ such that $n U \in \mathcal{F}$, then $\mathcal{F}$ is a Cauchy filter.

(2) For metrizable $E, E$ is an HM-space if and only if every bounded set is totally bounded.

(3) Examples of HM-spaces include the finite-dimensional spaces, FM-spaces, nuclear spaces, Silva spaces, and Schwarz spaces.

(4) $E$ is a complete $\mathrm{HM}$-space if and only if $E_{\mathrm{ns}}=E_{f}$. The space $\mathbb{R}^{\mathbb{N}}$ from 3.6 is a complete HM-space.

The proofs of the above remarks can be found in Henson and Moore [7] and [8].

Lemma 3.19 Suppose that $E$ is a complete HM-space and $f$ is smooth. Then $f$ is uniformly smooth at finite points.

Proof We will only show that $f$ is uniformly differentiable at finite points; the argument is the same for higher derivatives. Suppose $a \in \operatorname{in}\left(U^{*}\right) \cap E_{\mathrm{ns}}$ and $x \in E_{\mathrm{ns}}$. Then since $d f$ is continuous, we know that $d f(a)(x) \sim d f(\operatorname{st}(a))(\operatorname{st}(x))$, whence $d f(a)(x) \in F_{\mathrm{ns}} \subseteq F_{f}$. Now suppose that $\delta$ is a positive element of $\mu(\mathbb{R})$. Then, since $f^{[1]}$ and $d f$ are continuous, we have

$$
f^{[1]}(a, x, \delta) \sim f^{[1]}(\operatorname{st}(a), \operatorname{st}(x), 0)=d f(\operatorname{st}(a), \operatorname{st}(x)) \sim d f(a)(x) .
$$

\section{Finite Functions}

Throughout this section, $E$ and $F$ continue to denote locally convex spaces, but now $U$ denotes an open neighborhood of 0 in $E$. We still assume that $f: U \rightarrow F$ is any function.

Definition 4.1 We say that $f$ is a finite function if $f\left(U^{*} \cap E_{f}\right) \subseteq F_{f}$. 
In order for $f$ to induce a function on the nonstandard hulls, a necessary requirement is that $f$ be a finite function. Using Nelson's algorithm [12], one can give a standard translation of the notion that $f$ is a finite function. However, this equivalent standard condition is very complicated. Instead, we seek to prove that $f$ is finite under some natural assumptions.

Recall that a subset $B$ of a topological vector space $E$ is bounded if for any neighborhood $U$ of 0 in $E$, there exists $n$ such that $B \subseteq n U$. It is a well-known fact (see Henson and Moore [7, Theorem 2.1]) that $B$ is bounded if and only if $B^{*} \subseteq E_{f}$. We immediately get the following result.

Lemma 4.2 If $f(U)$ is a bounded subset of $F$, then $f$ is a finite function.

Recall that a subset $A$ of $E$ is symmetric if $A=-A$.

Lemma 4.3 Suppose $f: U \rightarrow F$ is uniformly continuous. Let $U_{1}$ be a symmetric open neighborhood of 0 in $E$ such that $U_{1}+U_{1} \subseteq U$. Then $f\left(U_{1}^{*} \cap E_{\mathrm{pns}}\right) \subseteq F_{f}$. In particular, if $E$ is an $H M$-space, then $f \mid U_{1}$ is a finite function.

Proof Let $x \in U_{1}^{*} \cap E_{\mathrm{pns}}$. We wish to show that $f(x) \in F_{f}$. Let $q$ be a continuous seminorm on $F$. Since $f$ is uniformly continuous, there is a symmetric open neighborhood $V$ of 0 such that whenever $a, b \in U$ are such that $a-b \in V$, then $q(f(a)-f(b))<1$. Since $x \in E_{\mathrm{pns}}$, we can find $y \in E$ such that $x-y \in U_{1}^{*} \cap V^{*}$. Then $y=x+(y-x) \in U^{*}$, whence $q(f(x)-f(y))<1$. Since $y$ is standard, $q(f(y)) \in \mathbb{R}_{f}$, whence $q(f(x)) \in \mathbb{R}_{f}$. Since $q$ was an arbitrary continuous seminorm on $F$, this shows that $f(x) \in F_{f}$.

We can improve Lemma 4.3 if we further assume that $U$ is convex, which is certainly the case for our applications. Recall that $f$ is said to be Lipschitz on large distances if for any continuous seminorm $r$ on $E$ and for any continuous seminorm $q$ on $F$, there is a continuous seminorm $p$ on $E$ so that $q\left(f\left(x_{1}\right)-f\left(x_{2}\right)\right) \leq p\left(x_{1}-x_{2}\right)$ for all $x_{1}, x_{2} \in U$ for which $r\left(x_{1}-x_{2}\right) \geq 1$. We will need the following fact.

Fact 4.4 (Corson and Klee [2]) A uniformly continuous mapping from a convex subset of a locally convex space $E$ into a locally convex space $F$ is Lipschitz on large distances.

Lemma 4.5 Suppose $f: U \rightarrow F$ is uniformly continuous and $U$ is convex. Then $f$ is a finite function. 
Proof Let $x \in U^{*} \cap E_{f}$ and let $q$ be a continuous seminorm on $F$. We wish to show that $q(f(x)) \in \mathbb{R}_{f}$. Clearly if $x \in \mu(E)$, then by continuity at 0 , we have $f(x) \in \mu(F)$. We thus may assume that $x \notin \mu(E)$. Choose a continuous seminorm $r$ on $E$ and $\epsilon \in \mathbb{R}^{>0}$ so that $r(x) \geq \epsilon$. By replacing $r$ by $\frac{1}{\epsilon} r$, we may assume that $r(x) \geq 1$. Let $p$ be a continuous seminorm on $E$ such that $q\left(f\left(x_{1}\right)-f\left(x_{2}\right)\right) \leq p\left(x_{1}-x_{2}\right)$ for all $x_{1}, x_{2} \in U$ satisfying $r\left(x_{1}-x_{2}\right) \geq 1$. Then since $r(x) \geq 1$, we have $q(f(x)-f(0)) \leq p(x) \in \mathbb{R}_{f}$, whence $q(f(x)) \in \mathbb{R}_{f}$.

A stronger assumption to impose on $f$ is that it is Lipschitz. Recall that $f$ is said to be Lipschitz if for every continuous seminorm $q$ on $F$, there is a continuous seminorm $p$ on $E$ so that $q\left(f\left(x_{1}\right)-f\left(x_{2}\right)\right) \leq p\left(x_{1}-x_{2}\right)$ for every $x_{1}, x_{2} \in U$.

Lemma 4.6 If $f$ is Lipschitz, then $f$ is a finite function.

Proof Let $x \in U^{*} \cap E_{f}$ and let $q$ be a continuous seminorm on $F$. Choose $p$ as in the definition of Lipschitz. Then $q(f(x)-f(0)) \leq p(x) \in \mathbb{R}_{f}$, which implies that $q(f(x)) \in \mathbb{R}_{f}$.

We end this section with a question. For $x_{0} \in E$ and $p$ a continuous seminorm on $E$, let $B_{1}^{p}\left(x_{0}\right)$ denote the set $\left\{x \in E \mid p\left(x-x_{0}\right)<1\right\}$. Say that $f$ is locally Lipschitz if for every $x_{0} \in U$ and every continuous seminorm $q$ on $F$, there is a continuous seminorm $p$ on $E$ such that $B_{1}^{p}\left(x_{0}\right) \subseteq U$ and $q(f(x)-f(y)) \leq p(x-y)$ for all $x, y \in B_{1}^{p}\left(x_{0}\right)$. One has the following result:

Fact 4.7 (Glockner [4, Lemma 1.9]) If $f$ is $C^{1}$, then $f$ is locally Lipschitz.

Question 4.8 It does not appear that assuming that $f$ is locally Lipschitz implies that $f$ is a finite function. Does the assumption that $f$ is uniformly $C^{1}$ at finite points (or even uniformly smooth at finite points) imply that $f$ is a finite function?

\section{Localizing Enlargeability}

In this section, we present our main theorem on localizing enlargeability and some of its corollaries. We first introduce some of the necessary definitions from locally convex Lie theory. 
Definition 5.1 A local Lie group is a tuple $\left(G, D, m_{G}, 1\right)$ such that $G$ is a smooth manifold modeled on a locally convex space, $D \subseteq G \times G$ is open, $m_{G}: D \rightarrow G$ (the product map) is smooth, and such that the following conditions hold:

- Suppose $x y$ and $y z$ are defined, that is $(x, y) \in D$ and $(y, z) \in D$. Then if one of the products $(x y) z$ or $x(y z)$ are defined, then so is the other and both products are equal;

- For each $x \in G$, we have $(x, 1) \in D$ and $(1, x) \in D$ and

$$
m_{G}(x, 1)=m_{G}(1, x)=x
$$

- For each $x \in G$, there is a unique $x^{-1} \in G$ such that $\left(x, x^{-1}\right),\left(x^{-1}, x\right) \in D$ and

$$
m_{G}\left(x, x^{-1}\right)=m_{G}\left(x^{-1}, x\right)=1 ;
$$

- The map $x \mapsto x^{-1}: G \rightarrow G$ is smooth;

- If $(x, y) \in D$, then $\left(y^{-1}, x^{-1}\right) \in D$.

Recall that a subset $A$ of a locally convex space $E$ is circular if $\lambda A \subseteq A$ for all $\lambda \in \mathbb{R}$ with $|\lambda| \leq 1$.

Definition 5.2 A Lie algebra $\mathfrak{g}$ is called locally exponential if there exists a circular, convex open 0 -neighborhood $U \subseteq \mathfrak{g}$ and an open subset $D \subseteq U \times U$ on which we have a smooth map

$$
m_{U}: D \rightarrow U, \quad(x, y) \mapsto x * y
$$

such that $\left(U, D, m_{U}, 0\right)$ is a local Lie group satisfying:

(E1) For $x \in U$ and $|t|,|s|,|t+s| \leq 1$, we have $(t x, s x) \in D$ and $t x * s x=(t+s) x$;

(E2) The second order term in the Taylor expansion of $m_{U}$ at $(0,0)$ is $[x, y]$.

The condition (E2) is only there to ensure that the Lie algebra of the local Lie group $\left(U, D, m_{U}, 0\right)$ is isomorphic to $\mathfrak{g}$.

Definition 5.3 Let $G$ be a Lie group with Lie algebra $\mathfrak{g}$.

(i) A smooth exponential map for $G$ is a smooth function $\exp _{G}: \mathfrak{g} \rightarrow G$ for which the curves $\gamma_{x}(t):=\exp _{G}(t x)(x \in \mathfrak{g})$ are 1-parameter subgroups of $G$ satisfying $\gamma_{x}^{\prime}(0)=x$. (Recall that a 1-parameter subgroup of $G$ is a continuous homomorphism $\mathbb{R} \rightarrow G$.) It is a fact that $G$ can possess at most one smooth exponential function; see the comments after Definition II.5.1 of the Neeb survey [11]. 
(ii) $G$ is said to be locally exponential if there exists a smooth exponential map $\exp _{G}$ for $G$, an open 0 -neighborhood $U \subseteq \mathfrak{g}$, and an open $e$-neighborhood $V \subseteq G$ such that $\exp _{G} \mid U$ is a diffeomorphism of $U$ onto $V$.

The above terminology is due to the fact that the Lie algebra of a locally exponential Lie group is a locally exponential Lie algebra; this follows from considering exponential coordinates. Thus a locally exponential Lie algebra is a natural candidate to be the Lie algebra of a locally exponential Lie group.

Definition 5.4 A locally exponential Lie algebra $\mathfrak{g}$ is said to be enlargeable if it is the Lie algebra of a locally exponential Lie group.

Pestov proves Theorem 1.1 in the following manner. First, he finds an internal Lie algebra $\mathfrak{h} \in \mathcal{H}^{*}$ such that $\mathfrak{g}$ embeds isometrically into $\widehat{\mathfrak{h}}$ as a closed subalgebra. By assumption, there is an internal Banach-Lie group $H$ for which $\mathfrak{h}$ is its Lie algebra. As mentioned in the Introduction, $\widehat{\mathfrak{h}}$ is a Banach-Lie algebra and Pestov shows that $\widehat{\mathfrak{h}}$ is enlargeable by constructing the nonstandard hull of $H$, which has $\widehat{\mathfrak{h}}$ as its Lie algebra. This finishes the proof of Theorem 1.1 as a closed Lie subalgebra of an enlargeable Banach-Lie algebra is enlargeable (see [15, page 22, item (***)]).

We now explain the set-up that allows us to pursue the above method of proof for the class of locally exponential Lie algebras. Suppose that $\mathfrak{g}$ is a locally exponential Lie algebra witnessed by $\left(U, D, m_{U}, 0\right)$. For $x, y \in U$, we sometimes write $x * y$ instead of $m_{U}(x, y)$. Suppose $\mathcal{H}$ is a directed family of closed subalgebras of $\mathfrak{g}$ and suppose that there exists an open, symmetric neighborhood $V \subseteq U$ of 0 in $\mathfrak{g}$ with $V \times V \subseteq D$ and such that

(1) $\bigcup \mathcal{H}$ is dense in $\mathfrak{g}$;

(2) for each $\mathfrak{h} \in \mathcal{H}$, there is a locally exponential Lie group $H$ such that $\operatorname{Lie}(H) \cong \mathfrak{h}$;

(3) for each $\mathfrak{h} \in \mathcal{H}$, if $H$ is a connected locally exponential Lie group such that $\operatorname{Lie}(H) \cong \mathfrak{h}$, then $\exp _{H} \mid V \cap \mathfrak{h}: V \cap \mathfrak{h} \rightarrow H$ is injective.

The preceding conditions are the direct analogues of Pestov's assumptions in the Banach setting. In order to make some of Pestov's arguments go through, we add two further conditions. First, we introduce some notation. For each $\mathfrak{h} \in \mathcal{H}$, let us fix a connected Lie group $H$ such that $\operatorname{Lie}(H) \cong \mathfrak{h}$ and let $W_{\mathfrak{h}}$ be an open symmetric neighborhood of $e$ in $H$ contained in $\exp _{H}(V)$. Let $D_{\mathfrak{h}}:=\left\{(x, y) \in \mathfrak{h} \times \mathfrak{h} \mid \exp _{H}(x) \cdot \exp _{H}(y) \subseteq W_{\mathfrak{h}}\right\}$, an open subset of $\mathfrak{h} \times \mathfrak{h}$. Define $*_{\mathfrak{h}}: D_{\mathfrak{h}} \rightarrow V \cap \mathfrak{h}$ by $x *_{\mathfrak{h}} y=\exp _{H}^{-1}\left(\exp _{H}(x) \cdot \exp _{H}(y)\right)$. Our new assumptions are that $W_{\mathfrak{h}}$ can be chosen so that there exists a continuous seminorm $p$ on $\mathfrak{g}$ for which: 
(4) $\{x \in \mathfrak{h} \mid p(x)<1\}^{\times 2} \subseteq D_{\mathfrak{h}}$;

(5) $\{x \in \mathfrak{g} \mid p(x)<1\}^{\times 2} \subseteq D$ and $m_{U} \mid\{x \in \mathfrak{g} \mid p(x)<1\}^{\times 2}$ is uniformly continuous.

We will need the following consequence of assumption (4).

Fact 5.5 (Glöckner and Neeb [5, Proposition 3.7.17]) Assumption (4) implies that for all $\mathfrak{h} \in \mathcal{H}$ and all $x, y \in \mathfrak{h}$ with $p(x), p(y)<1$, we have $x *_{\mathfrak{h}} y=m_{U}(x, y)$. (The statement of Proposition 3.7.17 in Glöckner and Neeb [5] is less precise than what we claim and one needs only to read the proof of that proposition to see that it yields this fact immediately.)

For the rest of this section, we fix $\mathfrak{h} \in \mathcal{H}^{*}$ and suppose $H$ is a corresponding internal connected locally exponential Lie group whose Lie algebra is $\mathfrak{h}$. For simplicity, let exp denote the exponential map for $H$. By the above fact, we can write $x * y$ instead of $x *_{\mathfrak{h}} y$ when $x, y \in \mathfrak{h}$ are such that $p(x)<1$ and $p(y)<1$.

The first step in constructing the nonstandard hull of $H$ is to define $H_{f}$. In the Banach setting of Pestov's paper, $H_{f}$ is defined in a certain technical fashion but is later shown to equal $\bigcup_{n} \exp (W)^{n}$, where $W$ is any ball around 0 in $\mathfrak{h}$ of finite, noninfinitesimal radius. It follows that $H_{f}=\bigcup_{n} \exp \left(\mathfrak{h}_{f}\right)^{n}$. Indeed, one inclusion is clear, for $W \subseteq \mathfrak{h}_{f}$. Now suppose $x \in \mathfrak{h}_{f}$. Choose $m$ so that $\frac{1}{m} x \in W$. Then $\exp (x)=\exp \left(\frac{1}{m} x\right)^{m} \in \exp (W)^{m}$, proving the other direction. I thus propose the following definition in the locally convex setting.

Definition 5.6 $H_{f}:=\bigcup_{n} \exp \left(\mathfrak{h}_{f}\right)^{n}$.

Lemma $5.7 H_{f}$ is a group.

Proof $H_{f}$ clearly contains $e$ and is closed under products. It remains to show that $H_{f}$ is closed under inverses; this follows from the fact that $\mathfrak{h}_{f}$ is closed under additive inverses and the fact that $\exp (-x)=\exp (x)^{-1}$.

In analogy with Pestov, we make the following definition.

Definition $5.8 \mu_{H}:=\exp \left(\mu_{\mathfrak{h}}\right)$.

The following lemma appears in Pestov's paper, where he uses facts about the $\mathrm{BCH}$ series in Banach-Lie algebras to reach this conclusion. We could not use such an argument and this is where assumptions (4) and (5) make their first appearance. 
Lemma 5.9 $\mu_{H}$ is a normal subgroup of $H_{f}$.

Proof First suppose that $x, y \in \mu_{H}$. Choose $x_{1}, y_{1} \in \mu_{\mathfrak{h}}$ such that $\exp \left(x_{1}\right)=x$ and $\exp \left(y_{1}\right)=y$. Using the continuity of $m_{U}$ and the fact that $(x, y) \in D_{\mathfrak{h}}$, we have $x_{1} * y_{1} \in \mu_{\mathfrak{h}}$ and thus $\exp \left(x_{1} * y_{1}\right) \in \mu_{H}$. But then

$$
x y=\exp \left(x_{1}\right) \exp \left(y_{1}\right)=\exp \left(x_{1} * y_{1}\right) \in \mu_{H} .
$$

Since $-x_{1} \in \mu_{\mathfrak{h}}$, we have $x^{-1}=\exp \left(-x_{1}\right) \in \mu_{H}$. Hence, $\mu_{H}$ is a subgroup of $H_{f}$.

Now suppose $y \in H_{f}$ and $x \in \mu_{H}$. We will show that $y x y^{-1} \in \mu_{H}$. Write $x=\exp \left(x_{1}\right)$, where $x_{1} \in \mu_{\mathfrak{h}}$. Let $z_{1}, \ldots, z_{n} \in \mathfrak{h}_{f}$ be such that $y=y_{1} \cdots y_{n}$, where $y_{i}:=\exp \left(z_{i}\right)$ for each $i \in\{1, \ldots, n\}$. Then

$$
y x y^{-1}=\exp \left(z_{1}\right) \cdots \exp \left(z_{n}\right) \exp \left(x_{1}\right) \exp \left(-z_{n}\right) \cdots \exp \left(-z_{1}\right) .
$$

By induction, it suffices to prove that, for all $z \in \mathfrak{h}_{f}$ and $w \in \mu_{\mathfrak{h}}$, we have

$$
\exp (z) \exp (w) \exp (-z) \in \mu_{H} .
$$

Choose a continuous seminorm $q \geq p$ on $\mathfrak{g}$ so that for all $x, y \in \mathfrak{g}$, if $q(x), q(y)<1$, then $p(x * y)<1$. Choose $m$ so that $q\left(\frac{1}{m} z\right)<1$. Let $w^{\prime} \in \mu_{\mathfrak{h}}$ be arbitrary. Then by uniform continuity of $m_{U}$, we know that $\frac{1}{m} z * w^{\prime} \sim \frac{1}{m} z$. Since $p\left(\frac{1}{m} z * w^{\prime}\right)<1$, another application of uniform continuity implies that $\left(\frac{1}{m} z * w^{\prime}\right) *\left(-\frac{1}{m} z\right) \sim\left(\frac{1}{m} z\right) *\left(-\frac{1}{m} z\right)=0$. Hence $\exp \left(\frac{1}{m} z\right) \exp \left(w^{\prime}\right) \exp \left(-\frac{1}{m} z\right) \in \mu_{H}$ for any $w^{\prime} \in \mu_{\mathfrak{h}}$. Since

$$
\exp (z) \exp (w) \exp (-z)=\exp \left(\frac{1}{m} z\right)^{m} \exp (w) \exp \left(-\frac{1}{m} z\right)^{m},
$$

we are finished with the proof of the lemma.

Definition 5.10 We set $\widehat{H}:=H_{f} / \mu_{H}$ and let $\pi_{H}: H_{f} \rightarrow \widehat{H}$ be the quotient map.

Lemma 5.11 Suppose $\pi_{\mathfrak{h}}(x)=\pi_{\mathfrak{h}}(y)$. Then $\pi_{H}(\exp x)=\pi_{H}(\exp y)$.

Proof Choose $m$ so that $p\left(\frac{1}{m} x\right), p\left(\frac{1}{m} y\right)<1$. By uniform continuity of $m_{U}$,

$$
\frac{1}{m} x *\left(-\frac{1}{m} y\right) \sim \frac{1}{m} y *\left(-\frac{1}{m} y\right)=0 .
$$

Thus $\exp \left(\frac{1}{m} x *-\frac{1}{m} y\right) \in \mu_{H}$, whence $\exp \left(\frac{1}{m} x\right) \exp \left(-\frac{1}{m} y\right) \in \mu_{H}$. Hence

$$
\begin{aligned}
\pi_{H}\left(\exp (x) \exp (y)^{-1}\right) & =\pi_{H}\left(\left(\exp \frac{1}{m} x\right)^{m-1}\left(\exp \frac{1}{m} x\right)\left(\exp \left(-\frac{1}{m} y\right)\right)\left(\exp \left(-\frac{1}{m} y\right)^{m-1}\right)\right. \\
& =\pi_{H}\left(\left(\exp \frac{1}{m} x\right)^{m-1}\left(\exp \left(-\frac{1}{m} y\right)\right)^{m-1}\right)
\end{aligned}
$$

Continuing in this fashion, one gets the desired result. 
The above lemma allows us to define a function $\widehat{\exp }: \widehat{\mathfrak{h}} \rightarrow \widehat{H}$ by

$$
\widehat{\exp }\left(x+\mu_{\mathfrak{h}}\right):=(\exp x) \mu_{H} .
$$

From now on, we will use the notation $\llbracket x \rrbracket$ for $x+\mu_{\mathfrak{h}}$ as introduced earlier in the paper.

Lemma 5.12 $\widehat{\exp }$ is injective on $\widehat{W}:=\{\llbracket x \rrbracket \mid \widehat{p}(\llbracket x \rrbracket)<1\}$.

Proof Suppose $\llbracket x_{1} \rrbracket, \llbracket x_{2} \rrbracket \in \widehat{W}$ are such that $\widehat{\exp }\left(\llbracket x_{1} \rrbracket\right)=\widehat{\exp }\left(\llbracket x_{2} \rrbracket\right)$. It follows that $\left(\exp x_{1}\right)\left(\exp x_{2}\right)^{-1} \in \mu_{H}$ and hence $\exp \left(x_{1} *\left(-x_{2}\right)\right) \in \mu_{H}$. Let $z \in \mu_{\mathfrak{h}}$ be such that $\exp \left(x_{1} *\left(-x_{2}\right)\right)=\exp z$. Since exp is injective on $V$, we have $x_{1} *\left(-x_{2}\right)=z$. But then, by uniform continuity of $m_{U}, x_{2} \sim\left(x_{1} *\left(-x_{2}\right)\right) * x_{2}=x_{1}$, that is $\llbracket x_{1} \rrbracket=\llbracket x_{2} \rrbracket$.

Remark 5.13 All that was used in the above construction of $\widehat{H}$ was that $m_{U}$ was $S$ continuous on pairs of points of $U^{*}$ with $p$-norm less than 1 which were finite, so the assumption that $m_{U}$ is uniformly differentiable at finite points allows those arguments to go through.

In addition to the conditions (1)-(5) we have imposed on the locally exponential Lie algebra $\mathfrak{g}$ and the family of closed subalgebras $\mathcal{H}$, we further assume the condition

(6) $m_{U}$ is uniformly smooth at finite points.

Note that condition (6), when combined with Remark 5.13 and Lemma 4.5, allows us to replace condition (5) with the following condition:

$\left(5^{\prime}\right) m_{U}$ is a finite function.

Under these conditions, we get a smooth map $\widehat{m_{U}}: \widehat{D} \rightarrow \widehat{\mathfrak{g}^{*}}$.

Lemma $5.14 \widehat{\mathfrak{h}}$ is a locally exponential Lie algebra.

Proof Let $\widehat{W}$ be as in Lemma 5.12. Consider the map

$$
m_{\widehat{\mathfrak{h}}}: \widehat{W} \times \widehat{W} \rightarrow \widehat{\mathfrak{h}}, \quad m_{\widehat{\mathfrak{h}}}(\llbracket x \rrbracket, \llbracket y \rrbracket):=\llbracket x * y \rrbracket .
$$

Note that $m_{\widehat{\mathfrak{h}}}=\widehat{m_{U}} \mid(\widehat{W} \times \widehat{W})$. Since $\widehat{\mathfrak{h}}$ is a complete subalgebra of $\widehat{\mathfrak{g}}^{*}$, it follows that $m_{\widehat{\mathfrak{h}}}$ is smooth. Let $D^{\prime}:=m_{\widehat{\mathfrak{h}}}^{-1}(\widehat{W})$. We claim that $\left(\widehat{W}, D^{\prime}, m_{\widehat{\mathfrak{h}}} \mid D^{\prime}, \llbracket 0 \rrbracket\right)$ witnesses that $\widehat{\mathfrak{h}}$ is a locally exponential Lie algebra.

It is clear that the above data yields a local group, and since multiplication and inversion are smooth (inversion is in fact linear), we have that $\left(\widehat{W}, D^{\prime}, m_{\widehat{\mathfrak{h}}} \mid D^{\prime}, \llbracket 0 \rrbracket\right)$ is a local Lie 
group. We now must verify conditions (E1) and (E2) of Definition 5.2. Towards proving (E1), suppose $\llbracket x \rrbracket \in \widehat{W}$ and $|t|,|s|,|t+s| \leq 1$. We need $(t \llbracket x \rrbracket, s \llbracket x \rrbracket) \in D^{\prime}$ and $m_{\widehat{\mathfrak{h}}}(t \llbracket x \rrbracket, s \llbracket x \rrbracket)=(t+s) \llbracket x \rrbracket$. Now since $\llbracket x \rrbracket \in \widehat{W}$, we know that $p(x)<1$, whence $(t x, s x) \in D_{\mathfrak{h}}$ and $t x * s x=(t+s) x$. It follows that $m_{\widehat{\mathfrak{h}}}(t \llbracket x \rrbracket, s \llbracket x \rrbracket)=(t+s) \llbracket x \rrbracket \in \widehat{W}$ and so $(t \llbracket x \rrbracket, s \llbracket x \rrbracket) \in D^{\prime}$.

Now suppose $h=\left(h_{1}, h_{2}\right) \in \mathfrak{g} \times \mathfrak{g}$. Since $d^{2} m_{U}(0,0)(h, h)=\left[h_{1}, h_{2}\right]$, Proposition 3.11 implies that

$$
d^{2} \widehat{m_{U}}(\llbracket 0 \rrbracket, \llbracket 0 \rrbracket)(\llbracket h \rrbracket, \llbracket h \rrbracket)=\llbracket\left[h_{1}, h_{2}\right] \rrbracket=\left[\llbracket h_{1} \rrbracket, \llbracket h_{2} \rrbracket\right] .
$$

It follows that the Lie algebra of the local group $\left(\widehat{W}, D^{\prime}, m_{\widehat{\mathfrak{h}}} \mid D^{\prime}, \llbracket 0 \rrbracket\right)$ is $\widehat{\mathfrak{h}}$. Hence (E2) holds and the proof of the lemma is finished.

Lemma 5.15 $\widehat{\mathfrak{h}}$ is enlargeable.

Proof By Lemma 5.12, $\widehat{\exp } \mid \widehat{W}$ is injective. It is also clear from the definitions that $\widehat{\exp }$ is a local group morphism when $\widehat{\mathfrak{h}}$ is endowed with the local group structure from Lemma 5.14. Let $\widehat{H_{1}}$ denote the subgroup of $\widehat{H}$ generated by $\widehat{\exp }(\widehat{W})$. Then Neeb [11, Corollary II.2.2] implies that $\widehat{H_{1}}$ carries the unique structure of a Lie group so that $\widehat{\exp } \mid \widehat{W}$ is a diffeomorphism onto an open subset of $\widehat{H_{1}}$. Then $\widehat{H_{1}}$ is a locally exponential Lie group with Lie algebra $\widehat{\mathfrak{h}}$, finishing the proof.

We are now ready to state our main theorem on localizing enlargeability.

Theorem 5.1 Suppose $\mathfrak{g}$ is a locally exponential Lie algebra and $\mathcal{H}$ is a family of closed subalgebras such that $\mathfrak{g}$ and $\mathcal{H}$ satisfy conditions (1)-(6) (or (5') instead of (5)). Then $\mathfrak{g}$ is enlargeable.

Proof As in Pestov [13], we get an internal $\mathfrak{h} \in \mathcal{H}^{*}$ such that the map $\iota: \mathfrak{g} \rightarrow \widehat{\mathfrak{g}}$ * actually takes values in $\widehat{\mathfrak{h}}$. For the sake of completeness, let us repeat how this argument goes. Let $X:=\bigcup \mathcal{H}$. Consider the following family of internal conditions on $A \in \mathcal{P}_{F}(X)^{*}$, the set of hyperfinite subsets of $X^{*}$ :

$$
C(g, p, n):=\left\{A \in \mathcal{P}_{F}(X)^{*} \mid \text { there exists } g^{\prime} \in A \text { such that } p\left(g-g^{\prime}\right)<\frac{1}{n}\right\},
$$

where $g$ ranges over $\mathfrak{g}$ and $p$ ranges over the set of continuous seminorms on $\mathfrak{g}$. Assumption (1) implies that for each $g \in \mathfrak{g}$ we have $\mu(g) \cap X^{*} \neq \emptyset$, whence the family of internal sets $C(g, p, n)$ has the finite intersection property. Hence, by saturation, 
there is an $A \in \mathcal{P}_{F}(X)^{*}$ belonging to each $C(g, p, n)$. Fix $g \in \mathfrak{g}$. We claim that $\mu(g) \cap A \neq \emptyset$. To see this, let

$$
B_{g}(p, n):=\left\{x \in A \mid p(g-x)<\frac{1}{n}\right\} .
$$

By the choice of $A$, each $B_{g}(p, n)$ is nonempty. Moreover, the family of internal sets $B_{g}(p, n)$, where $p$ ranges over the set of continuous seminorms on $\mathfrak{g}$, has the finite intersection property. Thus, by saturation, there is $x \in A$ which belongs to each $B_{g}(p, n)$, that is $x \in \mu(g) \cap A$. Since the family $\mathcal{H}$ is directed, there is $\mathfrak{h} \in \mathcal{H}^{*}$ such that $A \subseteq \mathfrak{h}$. This is the desired $\mathfrak{h}$.

Since $\iota: \mathfrak{g} \rightarrow \widehat{\mathfrak{h}}$ is an injective morphism of Lie algebras, we can infer that $\mathfrak{g}$ is enlargeable from the enlargeability of $\widehat{\mathfrak{h}}$ using Neeb [11, Corollary IV.4.10(2)].

We now mention a corollary of this theorem involving only standard notions. Say that a locally exponential Lie algebra $\mathfrak{g}$ is strong if there is a local group $\left(U, D, m_{U}, 0\right)$ witnessing that $\mathfrak{g}$ is a locally exponential Lie algebra for which $m_{U}$ is strongly smooth.

Corollary 5.16 If $\mathfrak{g}$ is a strong locally exponential Lie algebra with a family $\mathcal{H}$ of closed subalgebras satisfying conditions (1)-(4), then $\mathfrak{g}$ is enlargeable.

Proof This follows from the previous theorem, using Lemma 3.15, Lemma 3.17, and Lemma 4.5.

We can remove some of the assumptions on $\mathfrak{g}$ in Theorem 5.1 if $\mathfrak{g}$ is a complete HM-space.

Corollary 5.17 If $\mathfrak{g}$ is a locally exponential Lie algebra modeled on a complete HMspace with a family $\mathcal{H}$ of closed subalgebras satisfying conditions (1)-(4), then $\mathfrak{g}$ is enlargeable.

Proof It is clear from the continuity of $m_{U}$ and the fact that the finite points are all nearstandard that $m_{U}$ is a finite map. By Lemma 3.19, we know that $m_{U}$ is uniformly smooth at finite points.

Question 5.18 Corollary 5.16 and Corollary 5.17 would be exact analogs of Pestov's Theorem for certain classes of locally exponential Lie algebras if condition (4) were not needed. Can one eliminate assumption (4) in any of the above results?

Pestov draws the following corollary to his theorem. 
Corollary 5.19 (Pestov) If $\mathfrak{g}$ is a Banach-Lie algebra which contains a dense subalgebra in which every finitely generated subalgebra is finite-dimensional (or such that every finitely generated subalgebra is solvable), then $\mathfrak{g}$ is enlargeable.

Question 5.20 Even if one were able to obtain perfect analogs of Pestov's theorem for arbitrary locally exponential Lie algebras, would one be able to draw similar conclusions as in Corollary 5.19?

\section{References}

[1] W. Bertram, H. Glöckner, K.H. Neeb, Differential calculus over general base fields and rings, Expo. Math. 22(3)(2004), 213-282; doi:10.1016/S0723-0869(04)80006-9.

[2] H. Corson and V. Klee, Topological Classification of Convex Sets, Proc. Symp. Pure Math. VII (Convexity). Providence, R.I.: AMS 1963, 37-51; MR0161119.

[3] M. Davis, Applied Nonstandard Analysis, John Wiley and Sons Inc., 1977; MR0505473.

[4] H. Glöckner, Hölder Continuous Homomorphisms between InfiniteDimensional Lie Groups are Smooth, J. Funct. Anal. 228(2)(2005), 419-444; doi:10.1016/j.jfa.2005.06.023.

[5] H. Glöckner, K.H. Neeb, Infinite-dimensional Lie groups, Vol. I, Basic Theory and Main Examples, book in preparation.

[6] C.W. Henson, Foundations of Nonstandard Analysis: A Gentle Introduction to Nonstandard Extensions; in Nonstandard Analysis: Theory and Applications, 1-49, L. O. Arkeryd, N. J. Cutland, and C. W. Henson (eds.), NATO Science Series C, Springer, 2001.

[7] C.W. Henson and L.C. Moore, Jr., The Nonstandard Theory of Topological Vector Spaces, Trans. Amer. Math. Soc 172(1972), 405-435; MR0308722.

[8] C.W. Henson and L.C. Moore, Jr., Invariance of the Nonstandard Hulls of Locally Convex Spaces, Duke Math. J. 40(1973), 193-205; doi:10.1215/S0012-7094-73-040180 .

[9] M. Lazard, J. Tits, Domaines d'injectivité de l'application exponentielle, Topology 4 (1966), 315-322; doi:10.1016/0040-9383(66)90030-9.

[10] W.A.J. Luxemburg, A General Theory of Monads, in Internat. Sympos. Applications of Model Theory to Algebra, Analysis, and Probability (Pasadena, Calif., 1967), 18-89, Holt, Rinehart and Winston, New York, 1969; MR0244931. 
[11] K. H. Neeb, Towards a Lie Theory of Locally Convex Groups, Japan. J. Math. 1(2006), 291-468; doi:10.1007/s11537-006-0606-y.

[12] E. Nelson, Internal Set Theory: A New Approach to Nonstandard Analysis, Bull. Amer. Math. Soc. 83(1977), 1165-1198; doi:10.1090/S0002-9904-1977-14398-X

[13] V. Pestov, Nonstandard Hulls of Banach-Lie Groups and Algebras, Nova Journal of Algebra and Geom. 1 (1992), 371-384; MR1218361.

[14] K.D. Stroyan, Infinitesimal Calculus on Locally Convex Spaces: 1. Fundamentals, Trans. Amer. Math. Soc, 240(1978), 363-383; MR0493323.

[15] W.T. van Est and T.J. Korthagen, Non-enlargable Lie algebras, Nederl. Acad. Wetensch. Proc. A26(1964), 15-31; MR0160851.

Department of Mathematics, University of Illinois at Urbana-Champaign

1409 W. Green Street, Urbana, IL 61801, USA

igoldbr2@math.uiuc.edu

www . math. uiuc.edu/ igoldbr2

Received: 3 April $2008 \quad$ Revised: 2 April 2009 